\title{
PRODUCTION AND OPTIMIZATION OF BIODIESEL FROM PALM FATTY ACID DISTILLATE AND OLEIC ACID USING RESPONSE SURFACE METHODOLOGY APPROACH.
}

\author{
U, A. Maryam ${ }^{1}$. G. A Maspalma ${ }^{1}$, J. M Manu ${ }^{2}$ and M. B Hamid ${ }^{3}$, \\ ${ }^{1}$ Chemistry Unit, Department of Science Laboratory Technology, Adamawa State Polytechnic Yola, \\ Adamawa State, Nigeria. \\ ${ }^{2}$ Chemistry Unit, Department of Science Laboratory Technology, Moddibo Adama University of \\ Technology Yola, Adamawa State, Nigeria. \\ ${ }^{3}$ Biochemistry Unit, Department of Science Laboratory Technology, Adamawa State Polytechnic \\ Yola, Adamawa State, Nigeria. \\ *Corresponding author:agoddypalma@gmail.com:maryamusman28@gmail.com \\ Received 18 January 2020; accepted 28 January 2020, published online 30 January 2021
}

\begin{abstract}
In this study, a mixed feed of Palm fatty acid distillate (PFAD)/ oleic acid was used to produced biodiesel catalyzed by sulfonated carbon. The effect of three process variables i.e. methanol - to PFAD/oleic acid molar ratio, catalyst loading and reaction time on the yield of biodiesel produced was studied using response surface methodology (RSM) based on Box-Behnken design (BBD). Optimum reaction conditions were obtained at 1:7 methanol- to-PFAD/oleic acid molar ratio, 15 wt.\% catalyst loading and $5 \mathrm{~h}$ reaction time. The predicted biodiesel yield was $96 \%$ under the optimal conditions. From the results obtained, it can be deduced that PFAD/ oleic acid has a high potential as an inedible feedstock to produce low cost biodiesel, and the method may be useful for industrial process optimization.
\end{abstract}

Keywords: Palm fatty acid distillate, oleic acid, Optimization. Response Surface Methodology.

\section{INTRODUCTION}

The depletion in fossil fuels, environmental pollution, and high cost in prices of petroleum products has led scientists to look for alternative fuels such as biofuels, obtained from sources that can be renewed [11]. Biodiesel, as a biofuel, has the potential to be used as a substitute to diesel fuel because it is biodegradable, non-toxic, inflammable,

produces less emission of carbon monoxide, sulphur dioxide, and unburnt hydrocarbons

when compared to diesel fuel [4]. Itis a fuel obtained from vegetable oils or fats and methanol [1]. However, the use of edible oils is not practical, as this brings shortage in food, and a hike in food prices. This limitation can be overcome by the use of inedible feed stocks all of which are low-cost feed stocks. PFAD and oleic acid are the most promising alternative low cost feed stocks as they are abundant, cheap and readily available. However, due to their high free fatty acids content, a suitable catalyst is required for their esterification and transesterification. Solid acid catalysts are promising heterogeneous catalysts that have a great influence in reducing the cost of biodiesel production as a result of its effectiveness in promoting the conversion of FFA to biodiesel (FAME). They are insensitive to FFA, which makes them economically viable [12]. The ease in which they are separated from the product at the end of the reaction also adds greatly to its advantages. Unlike homogeneous catalysts which pose a threat economically, and environmentally. Therefore, using oleic acid, PFAD/oleic acid mixed feed and sulfonated carbon based catalyst as a heterogeneous acid catalyst generally describes esterification and transesterification reaction for FFAs to produce biodiesel.

In biodiesel synthesis, the output depends on the interaction between variables during the reaction. The variables commonly varied for biodiesel synthesis include molar ratio of oil to alcohol, time, temperature, and catalyst loading. Determining the optimum conditions in biodiesel production brings about a better utilization of resources. The conventional method of process optimization considers the effect of one variable at a time, which is expensive and time consuming. To overcome this problem, design of experiment (DOE) is used to study multiple variables in the same experiment. Response Surface Methodology (RSM) using Box- Behnken Design (BBD) is 
one of the suitable tools for optimizing biodiesel production process. It is a statistical tool that is used to predict a mathematical model based on the fitness of experimental data obtained in relation to experimental design [3].

The reaction conditions for biodiesel production using different catalysts have been optimized using RSM by many authors [6], [11]. However, little or no work has been carried out on the production of biodiesel from PFAD/oleic acid mixed feed using sulfonated carbon catalyst. This study is therefore aimed at investigating the activity of the catalyst on the production of biodiesel from PFAD/oleic acid and optimization of the esterification/transesterification of PFAD/oleic acid mixed feed using Response Surface Methodology (RSM).

\section{EXPERIMENTAL}

\section{Chemical and Reagents}

All reagents were used without any purification. Palm fatty acid Distillate with high FFA content were supplied by Johor Palm oil refinery, SDN BHD, Malaysia. Oleic acid was obtained from Chempur, Germany. Sulfuric acid (95-97\% purity), Ethanol (96\% purity), silicon oil, and methanol (> $99.9 \%$ purity) were obtained from Q Rec, New Zealand. Glycerol (>99.5\% purity) was purchased from Sigma Aldrich, USA. And other reagents such as methyl oleate standard, methyl palmitate standard with purity of $97 \%$, and phenolphthalein were obtained from Merck, Germany. All the reagents used for the study were of analytical grade.

\section{Preparation of Catalyst}

The sulfonated carbon was prepared according to the method proposed by [5]. 10g glycerol was mixed with $20 \mathrm{~g}$ concentrated sulfuric acid in a $500 \mathrm{ml}$ beaker, and gently heated on a hotplate from $25^{\circ} \mathrm{C}$ to $220^{\circ} \mathrm{C}$ The temperature was kept constant for $20 \mathrm{~min}$ till it stopped foaming. The product obtained was washed under agitation with hot water until it became neutral to $\mathrm{pH}$. The product (sulfonated carbon) was then filtered and dried at $110^{\circ} \mathrm{C}$ for $2 \mathrm{hrs}$ in an oven so as ensure it is moisture free.

\section{Esterification of PFAD/Oleic acid}

The simultaneous esterification of PFAD/oleic acid was carried out. A mixture of PFAD/oleic acid was placed in a $100 \mathrm{ml}$ three-necked flask that was connected with a reflux condenser, a thermometer and a stopper. PFAD/oleic acid: methanol molar ratio (1:3-1:11), catalyst loading (5-30 wt \%), reaction temperature $\left(65^{\circ} \mathrm{C}\right)$, and reaction time $(1-6 \mathrm{~h})$ were varied accordingly. PFAD/oleic acid, methanol, and the sulfonated carbon catalyst were put into the flask and heated at $65^{\circ} \mathrm{C}$ under stirring for $5 \mathrm{~h}$. After the reaction reached completion, the catalyst was filtered off, and the product obtained was poured into a separating funnel. The upper layer consists of Fatty acid methyi esters, which was washed with distilled water to remove any impurities present, dried in an oven and taken for further analysis.

\section{Design of Experiment for PFAD/Oleic Acid Mixed Feed}

A Box-Behnken experimental with three variables was used to investigate the response pattern and determine its combination of the variables. The range of the variables were determined from the parameter study done on PFAD/oleic acid mixed feed. And the methanol to PFAD/oleic acid molar ratio (A), catalyst loading (B), and time (C) were taken as the independent variables selected for the optimization. The coded and uncoded levels of the independent variables used for the esterification are given in Table 1.

Table 1: Experimental range and factor levels of process variables tested in the esterification of PFAD/oleic acid.

\begin{tabular}{lcccc}
\hline \multicolumn{1}{c}{ Factors } & Symbol & \multicolumn{3}{c}{ Range and levels } \\
\hline Methanol to PFAD/oleic acid & A & $\mathbf{- 1}$ & $\mathbf{0}$ & $\mathbf{+ 1}$ \\
Catalyst loading (wt \%) & B & 5 & 7 & 9 \\
Reaction Time (h) & C & 10 & 15 & 20 \\
& & 3 & 4.5 & 6 \\
\hline
\end{tabular}




\section{PFAD/Oleic acid methyl ester analysis}

The quantification was conducted using a gas chromatograph equipped with a flame ionization detector (GC-FID) (Perkin Elmer, USA). The GC was fitted with a DB-Wax column $(30 \mathrm{~m} \times 0.53 \mathrm{~mm}, 0.5 \mu \mathrm{m})$. Conditions used were as follows; injector temperature, $250^{\circ} \mathrm{C}$; detector temperature, $270^{\circ} \mathrm{C}$; initial oven temperature, $60^{\circ} \mathrm{C}$ with holding time of 1 min; ramp rate, $25^{\circ} \mathrm{C} / \mathrm{min}$; and final oven temperature, $220^{\circ} \mathrm{C}$ with holding time of 10 min. And nitrogen was used as the carrier gas for the GC.

The prepared biodiesel (250mg) was dissolved in $5 \mathrm{ml} \mathrm{of} 5 \mathrm{mg} / \mathrm{mL}$ of methanol for the gas chromatography analysis. Methyl oleate and methyl palmitate were used as the internal standard to quantify the ester content in the biodiesel. The methyl ester content was quantified by comparing the peak area between the samples and the standard compounds. And the yield was calculated from the relation below:

$$
\text { FAME Yield, Y1 (\%) = C } x \frac{\text { Weight of FAME produced }}{\text { Weight of Feed }} \times 100 \%
$$

Where $\mathrm{C}$ is the Fame Content.

\section{PFAD/ Oleic Acid Conversion.}

To determine the PFAD/oleic acid conversion, $0.1 \mathrm{~g}$ of sample was mixed with $10 \mathrm{ml}$ of isopropanol as solvent and phenolphthalein as indicator in a beaker, and was shaken to ensure homogeneous mixing of the reactants. This mixture was then titrated with $0.1 \mathrm{~N} \mathrm{KOH}$ and Acid value $\left(\mathrm{mg} \frac{\mathrm{KOH}}{\mathrm{g}}\right)=\frac{(\mathrm{Vf}-\mathrm{Vi}) \times \mathrm{N} \times 56.1)}{\mathrm{W}} \times 100 \%$

Where:

$\mathrm{V}_{\mathrm{f}}=$ Final volume of standard alkali used in the titration (ml).

$\mathrm{V}_{\mathrm{i}}=$ Initial volume of standard alkali used in the titration $(\mathrm{ml})$.

$\mathrm{N}=$ Normality of standard alkali.

$$
\text { Conversion, } \%=\frac{\mathrm{AVf}-\mathrm{AVp}}{\mathrm{AVf}} \times 100 \% \text { (Eq. 2.3) }
$$

Where $A V_{f}$ and $A V_{p}$ are acid values of the feedstock's and their products (FAME) respectively

\section{RESULTS AND DISCUSSION PFAD/ Oleic Acid Esterification Using RSM (BBD), Model Fitting and Analysis of Variance (ANOVA).}

Response surface methodology based on BoxBehnken Design was applied to predict the best performance conditions for the esterification and transesterification of PFAD/oleic acid mixed feed. It was employed to model the conversion and yield of PFAD/oleic acid mixed feed using the shaken vigorously. The titration was stopped when the clear mixture turned to a permanent pink colour indicating that the end point had been reached. The amount of $\mathrm{KOH}$ that was used for the titration was recorded and then used to determine the acid values of the samples and their products, which were calculated using Equation 2.2 [2].

(Eq. 2.2)

$\mathrm{W}=$ Mass of Sample $(\mathrm{g})$.

The conversion of PFAD/oleic acid was determined based on the acid value of PFAD/oleic acid, and also the final product. The conversion was calculated using Equation 2.3. Potassium hydroxide $(\mathrm{KOH})$ titration was carried out to determine the acid value.

synthesized sulfonated carbon catalyst. The variables used were molar ratio (1:5- 1:9), catalyst loading (10-20), and reaction time (36h). A total of 17 experiments were carried out altogether, and the results obtained are shown in Tables 3.1a and b. The highest conversion obtained was $98 \%$. While for yield, $94 \%$ and 96\% for methyl oleate and methyl palmitate were obtained respectively.

On completion of the esterification/transesterification, the catalyst was filtered off and the product was allowed to cool down to room temperature. Two layers were obtained.The upper layer containing FAME was separated and taken for analysis. 
J. Chem. Soc. Nigeria, Vol. 46, No.1, pp 0036 - 0048 [2021]

Table2: Experimental designs for PFAD/oleic acid

\begin{tabular}{l|c|c|c}
\hline Run & $\begin{array}{c}\text { A } \\
(\mathrm{mol} / \mathrm{mol})\end{array}$ & $\begin{array}{c}\mathrm{B} \\
(\mathrm{wt} \%)\end{array}$ & $\begin{array}{c}\mathrm{C} \\
(\mathrm{h})\end{array}$ \\
\hline 1 & 5 & 20 & 4.50 \\
2 & 7 & 20 & 3.00 \\
3 & 7 & 15 & 4.50 \\
4 & 5 & 15 & 3.00 \\
5 & 9 & 15 & 6.00 \\
6 & 7 & 15 & 4.50 \\
7 & 7 & 10 & 6.00 \\
8 & 5 & 15 & 6.00 \\
9 & 7 & 15 & 4.50 \\
10 & 7 & 15 & 4.50 \\
11 & 7 & 10 & 3.00 \\
12 & 9 & 10 & 4.50 \\
13 & 7 & 20 & 6.00 \\
14 & 7 & 15 & 4.50 \\
16 & 9 & 20 & 4.50 \\
17 & 9 & 15 & 3.00 \\
\hline
\end{tabular}

Table 3: Experimental and predicted responses for PFAD/oleic acid using Response Surface Methodology (BBD).

\begin{tabular}{|c|c|c|c|c|c|c|}
\hline \multicolumn{3}{|c|}{ Run } & $\begin{array}{l}\text { onversion (\%) } \\
\text { Methyl Oleate }\end{array}$ & \multicolumn{2}{|c|}{$\begin{array}{l}\text { Yield }(\%) \\
\text { Methyl Palmitate }\end{array}$} & \\
\hline $\mathrm{S} / \mathrm{N}$ & Experimental & Predicted & Experimental & Predicted & Experimenta & Predicted \\
\hline 1 & & 87.12 & 90 & 83.13 & 92 & 85.12 \\
\hline 2 & 98 & 93.36 & 94 & 89.38 & 96 & 91.37 \\
\hline 3 & 97 & 94.62 & 93 & 90.63 & 95 & 92.62 \\
\hline 4 & 92 & 88.87 & 88 & 84.88 & 90 & 86.87 \\
\hline 5 & 90 & 91.50 & 86 & 87.50 & 88 & 89.50 \\
\hline 6 & 98 & 94.25 & 94 & 90.25 & 96 & 92.25 \\
\hline 7 & 95 & 92.75 & 91 & 88.75 & 93 & 90.75 \\
\hline 8 & 93 & 90.50 & 89 & 86.50 & 91 & 88.50 \\
\hline 9 & 98 & 94.37 & 94 & 90.38 & 96 & 92.37 \\
\hline 10 & 98 & 97.87 & 94 & 93.88 & 96 & 95.88 \\
\hline 11 & 94 & 95.12 & 90 & 91.13 & 92 & 93.12 \\
\hline 12 & 94 & 94.62 & 90 & 90.63 & 92 & 92.63 \\
\hline 13 & 95 & 97.80 & 91 & 93.80 & 93 & 95.80 \\
\hline 14 & 98 & 97.80 & 94 & 93.80 & 96 & 95.80 \\
\hline 15 & 87 & 97.80 & 83 & 93.80 & 85 & 95.80 \\
\hline 16 & 89 & 97.80 & 85 & 93.80 & 87 & 95.80 \\
\hline 17 & 94 & 97.80 & 90 & 93.80 & 92 & 95.80 \\
\hline
\end{tabular}


Table 4: Statistical summary for the three Models

\begin{tabular}{l|l|l|l}
\hline Sources & $\begin{array}{l}\text { PFAD/Oleic Acid } \\
\text { conversion }\end{array}$ & Methyl Oleate yield & $\begin{array}{l}\text { Methyl Palmitate } \\
\text { yield }\end{array}$ \\
\hline Standard Deviation & 0.60 & 0.60 & 0.60 \\
Mean & 94.35 & 92.35 & 92.35 \\
coefficient of variation & 0.64 & 0.65 & 0.65 \\
PRESS & 29.25 & 29.25 & 29.25 \\
R-Squared & $0 / 9861$ & 0.9861 & 0.9861 \\
Adjusted R- Squared & 0.9683 & 0.9683 & 0.9683 \\
Predicted R- Squared & 0.8409 & 0.8409 & 0.8409 \\
Adequate Precision & 23.233 & 23.333 & 23.333 \\
\hline
\end{tabular}

\section{Regression Model and Analysis of Variance for the Responses.}

Response surface methodology with Box Behnken Design (BBD) was employed to study the interaction between the responses and operating parameters that were used in the production of biodiesel from PFAD/oleic acid mixed feed using sulfonated carbon catalyst. A second order polynomial was used to show the regression models relating PFAD/oleic acid mixed feed conversion $\left(Y_{1}\right)$, methyl oleate yield $\left(Y_{2}\right)$, and methyl palmitate $\left(Y_{3}\right)$. These and the coded factors are shown in Eqs $3.1-$ 3.3. The selected models consisted of three levels $(-1,0,+1)$ and the data was evaluated statistical analysis parameters such as the $P-$ value, $F$-value, degrees of freedom $(D F)$, coefficient variation $(C V)$, and so on.

$\mathrm{Y}_{1}=+97.80+0.13 \mathrm{~A}+0.75 \mathrm{~B}-0.63 \mathrm{C}-$ $3.00 \mathrm{AB}-1.25 \mathrm{AC}-1.00 \mathrm{BC}-5.03 \mathrm{~A}^{2}-1.78 \mathrm{~B}^{2}$ $-0.52 \mathrm{C}^{2}$ (Eq.3.1)

$\mathrm{Y}_{2}=+93.80+0.12 \mathrm{~A}+0.57 \mathrm{~B}-0.63 \mathrm{C}-$ $3.00 \mathrm{AB}-1.25 \mathrm{AC}-1.00 \mathrm{BC}-5.02 \mathrm{~A}^{2}-1.77 \mathrm{~B}^{2}-$ $0.53 \mathrm{C}^{2} \quad(\mathrm{Eq} 3.2)$

$\mathrm{Y}_{3}=+95.80+0.13 \mathrm{~A}+0.75 \mathrm{~B}-0.63 \mathrm{C}-$ $3.00 \mathrm{AB}-1.25 \mathrm{AC}-1.00 \mathrm{BC}-5.03 \mathrm{~A}^{2}-1.78 \mathrm{~B}^{2}-$ $0.52 \mathrm{C}^{2}$

(Eq 3.3)

Where $\mathrm{Y}_{1}, \mathrm{Y}_{2}$, and $\mathrm{Y}_{3}$ are the responses, conversion, methyl oleate and

The diagnostic plots generated by the RSM showing the predicted versus the actual data and the residual versus the predicted data are represented in Figs 1-3.They show the predicted values close to the responses of the actual experiments implying that the errors methyl palmitate respectively. A is molar ratio of methanol to PFAD/oleic acid mixed feed, B is catalyst loading, and $\mathrm{C}$ is reaction time and are the coded factors.

The Statiscal summary for the Analysis of Variance (ANOVA) generated for this study is shown in Tables 4.. It was used to study the significance and fitness of the quadratic models and their responses in relation to the interaction of the variables in the reaction. From the tables, it can be seen that the variables had affected the responses of the models significantly. The $\mathrm{P}$ - values obtained were less than 0.05 indicating the significance of the model terms[13]. And the insignificant lack of fit models in the tables indicate that the models were fit.

From Tables 4 , the coefficient of $\mathrm{R}^{2}$ were 0.9861 for PFAD/oleic acid conversion, methyl oleate and methyl palmitate. Thereby, showing the adequacy of the model. And the 'Pred. R-squared' (0.8409) obtained were in confirmation with the 'Adj R-squared' (0.9683). Furthermore, 'Adeq. Precision' ratios of 23.223 were obtained indicating adequate signals. Low values of coefficient of variation (CV) for conversion of (0.64), methyl oleate $(0.65)$ and methyl palmitate $(0.65)$ were also obtained. This indicates that the high degree of precision and experimental value were in good range [8]

were all and well distributed [8]. This is also in agreement with the adjusted $\mathrm{R}^{2}$ values of 0.9683, which indicates the models had variabilities of $96.83 \%$. 
J. Chem. Soc. Nigeria, Vol. 46, No.1, pp 0036 - 0048 [2021]

(a)

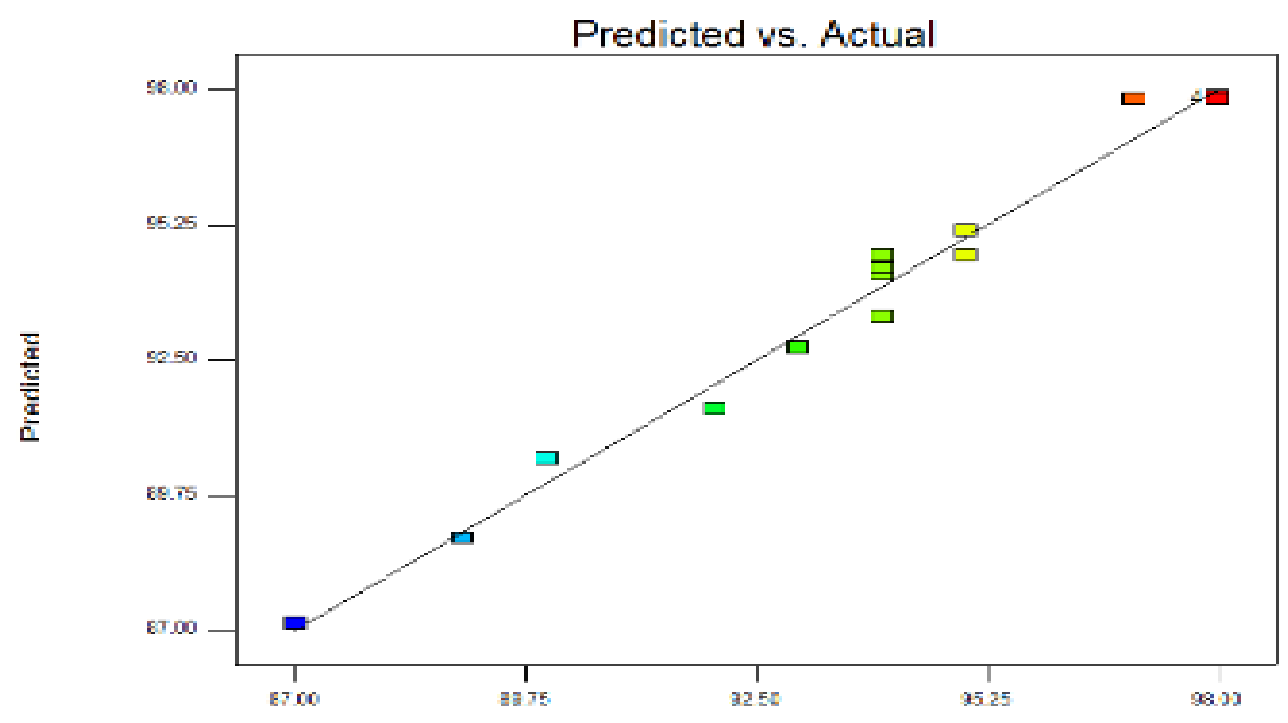

(b)

Actual

Residuals vs. Predicted

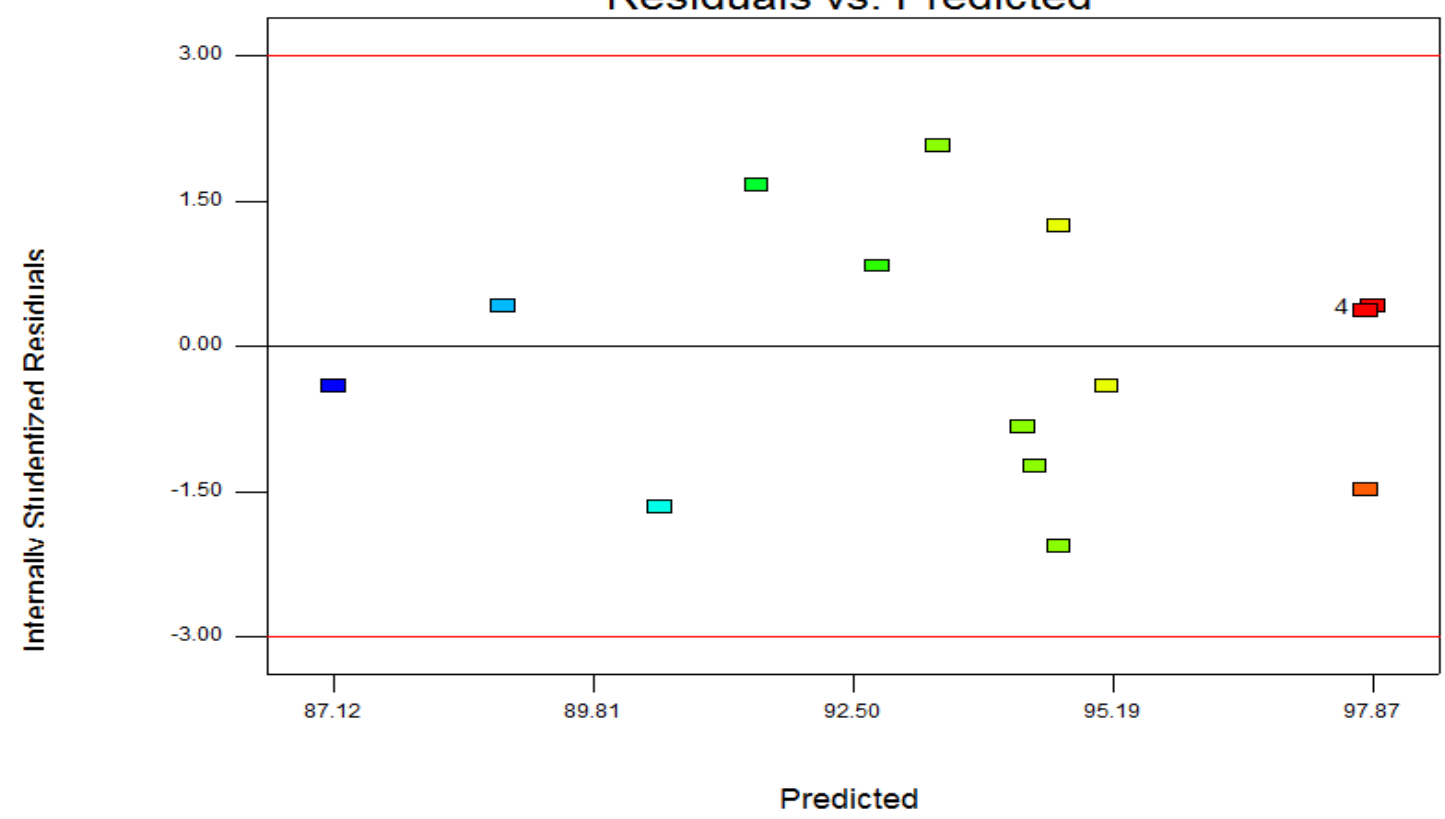

Fig 1: Diagnostic plots for conversion of PFAD/oleic acid mixed feed showing (a) Predicted vs. Actual and (b) Residual vs. Predicted 
J. Chem. Soc. Nigeria, Vol. 46, No.1, pp 0036 - 0048 [2021]

(a)
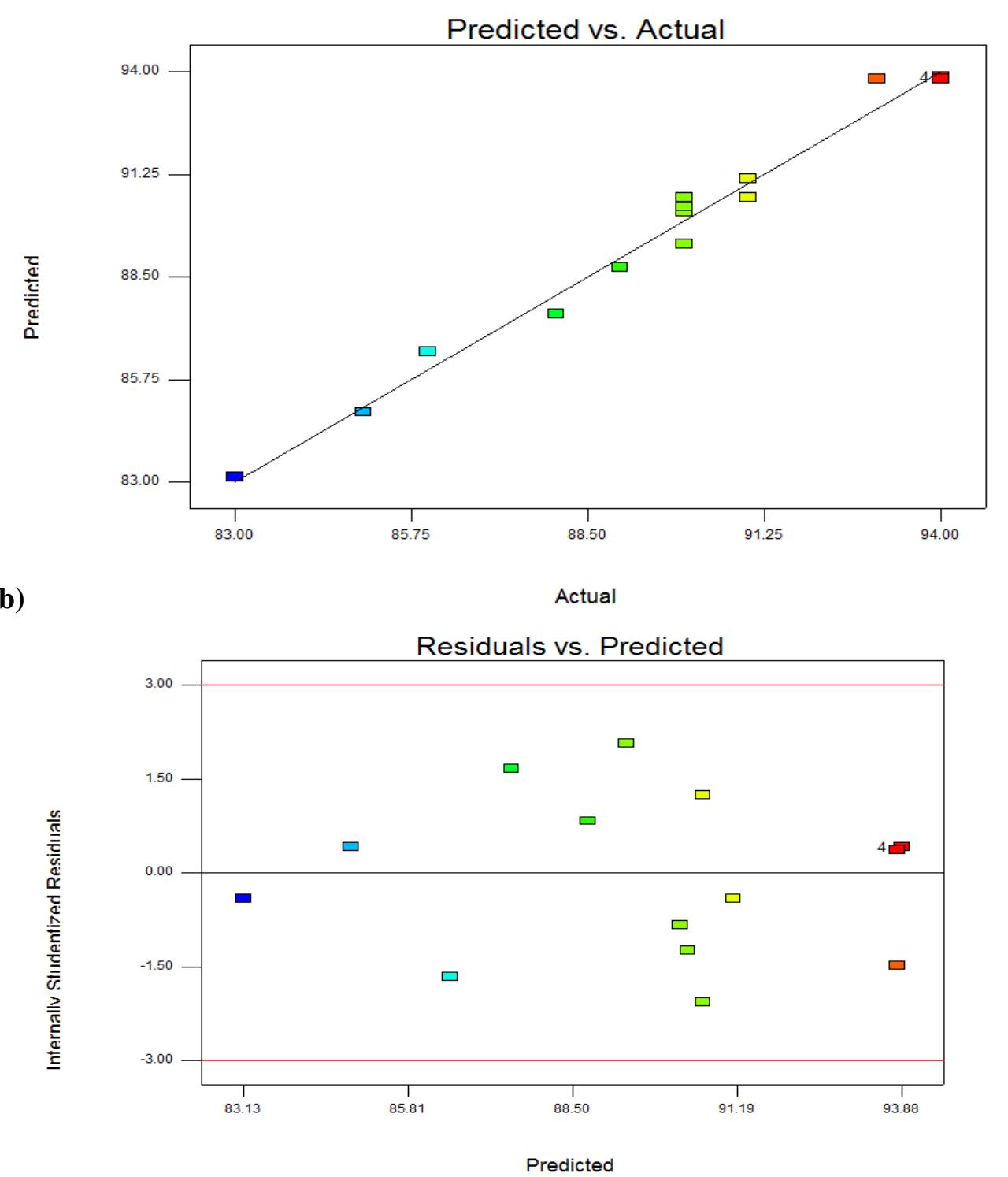

Fig 2: Diagnostic plots for methyl oleate yield showingresponsesfor a) Predicted vs. Actual and b) Residual vs. Predicted. 
J. Chem. Soc. Nigeria, Vol. 46, No.1, pp 0036 - 0048 [2021]

(a)

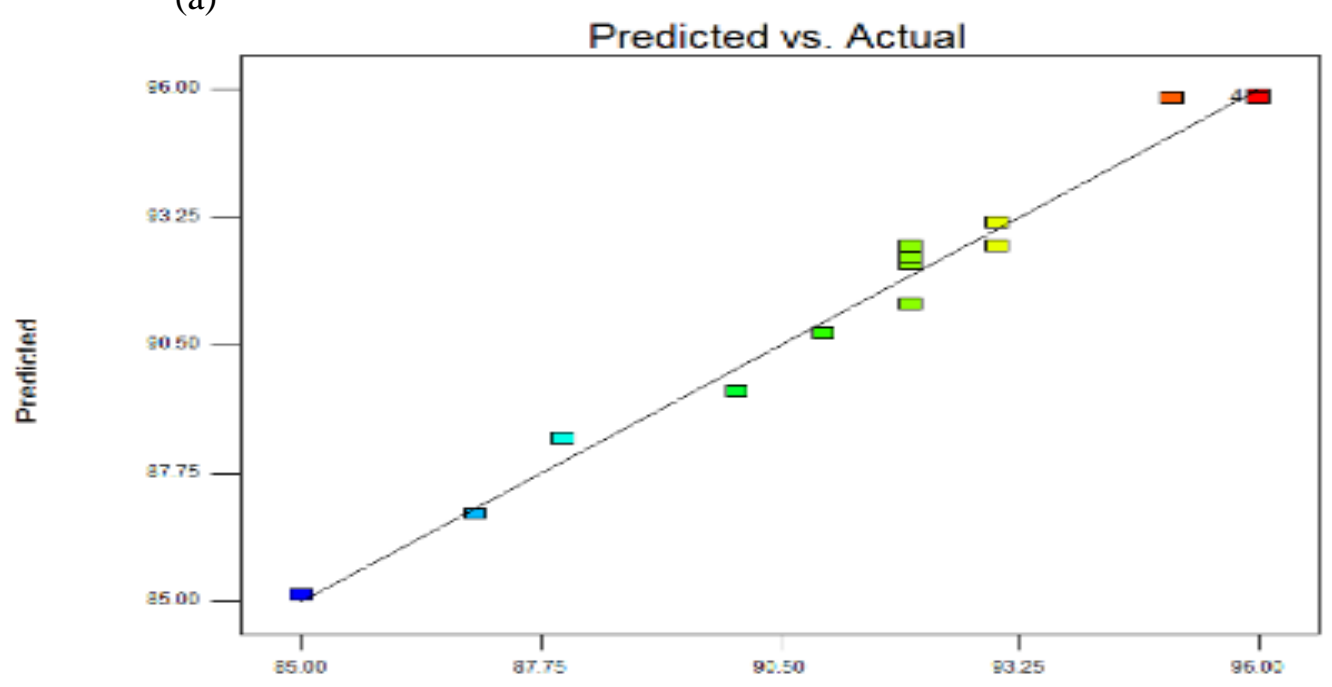

(b)

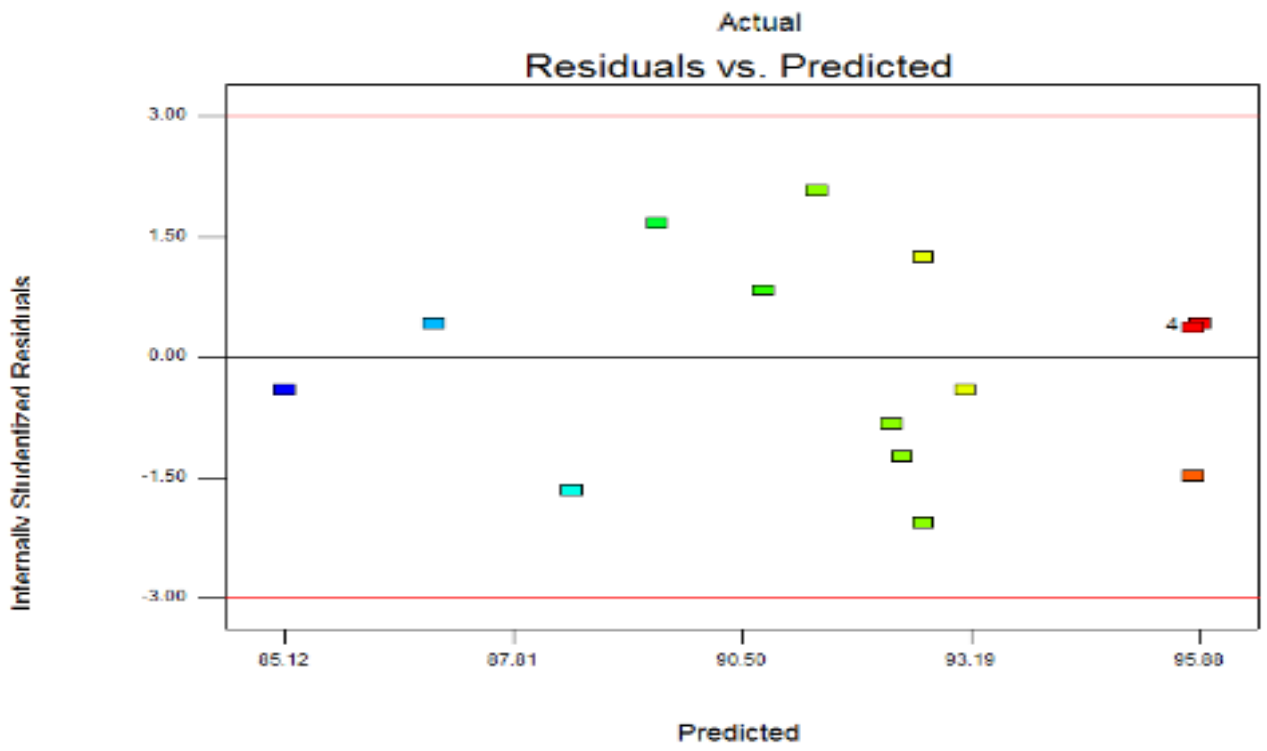

Fig 3: Diagnostic plots for methyl palmitate yield showingresponsesfor a) Predicted vs. Actual and b) Residual vs. Predicted.

The effects of molar ratio of methanol: PFAD/oleic acid, catalyst loading and reaction time are shown in fig 3 . It can be seen that catalyst loading and reaction time were the most significant. In the conversion of PFAD/oleic acid, methyl oleate and also methyl palmitate, there was increase as all the variables were increased. 
J. Chem. Soc. Nigeria, Vol. 46, No.1, pp 0036 - 0048 [2021]

(A)

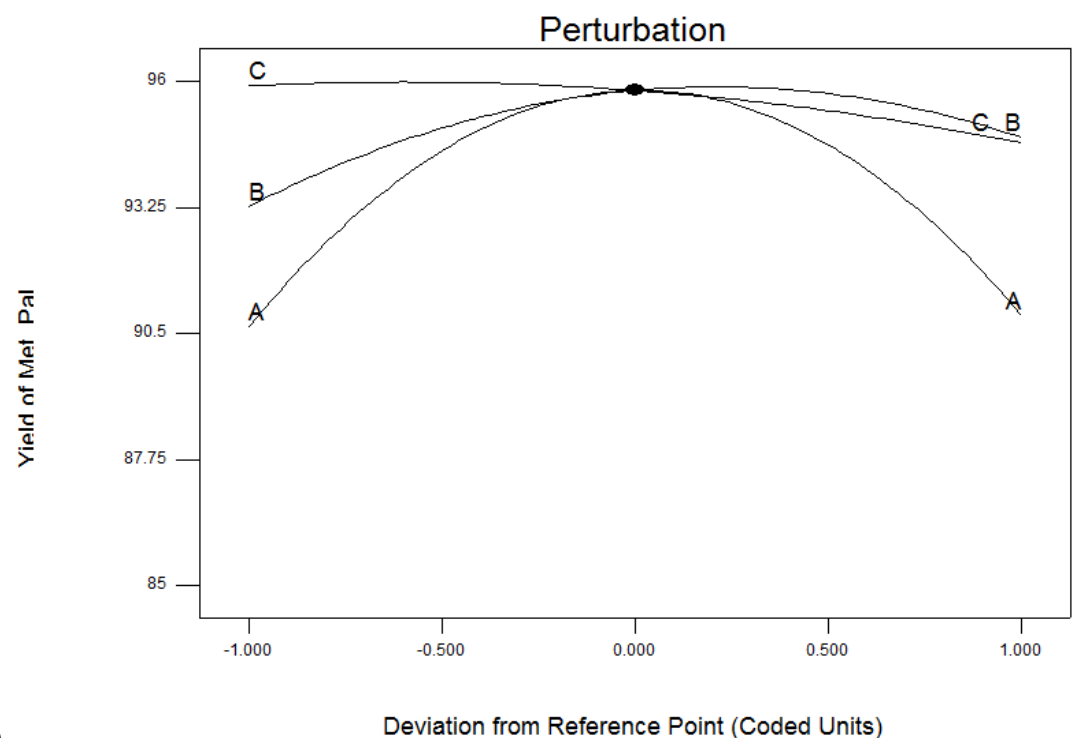

(B)

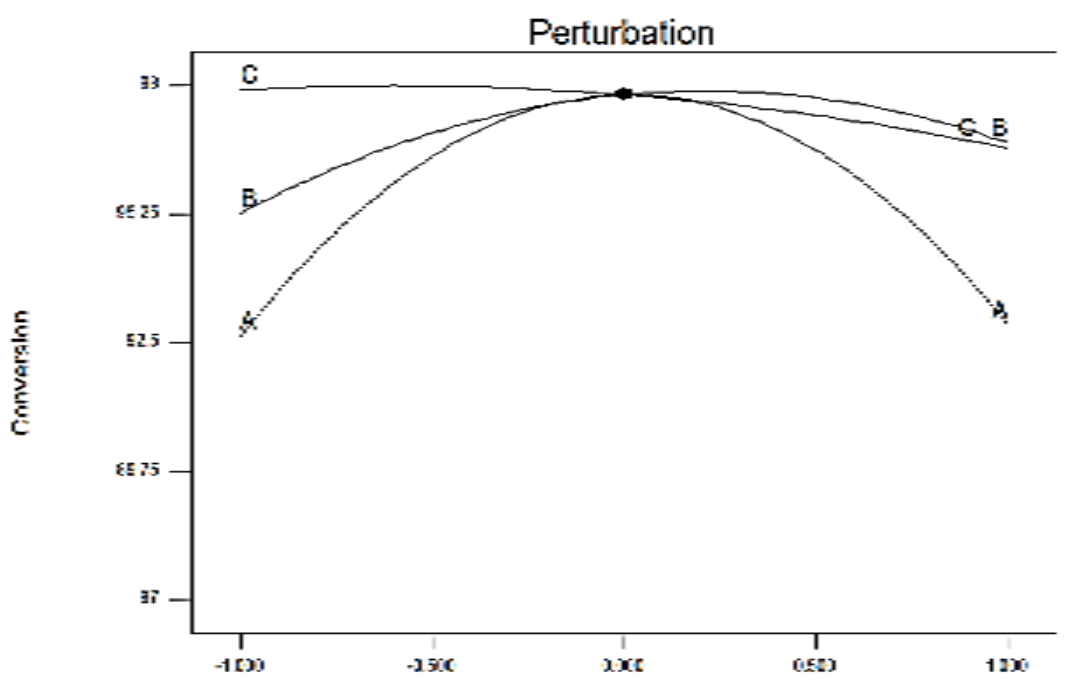

(C)

Desiajicn Trem Fieference Pcirt iCocied Unirs:

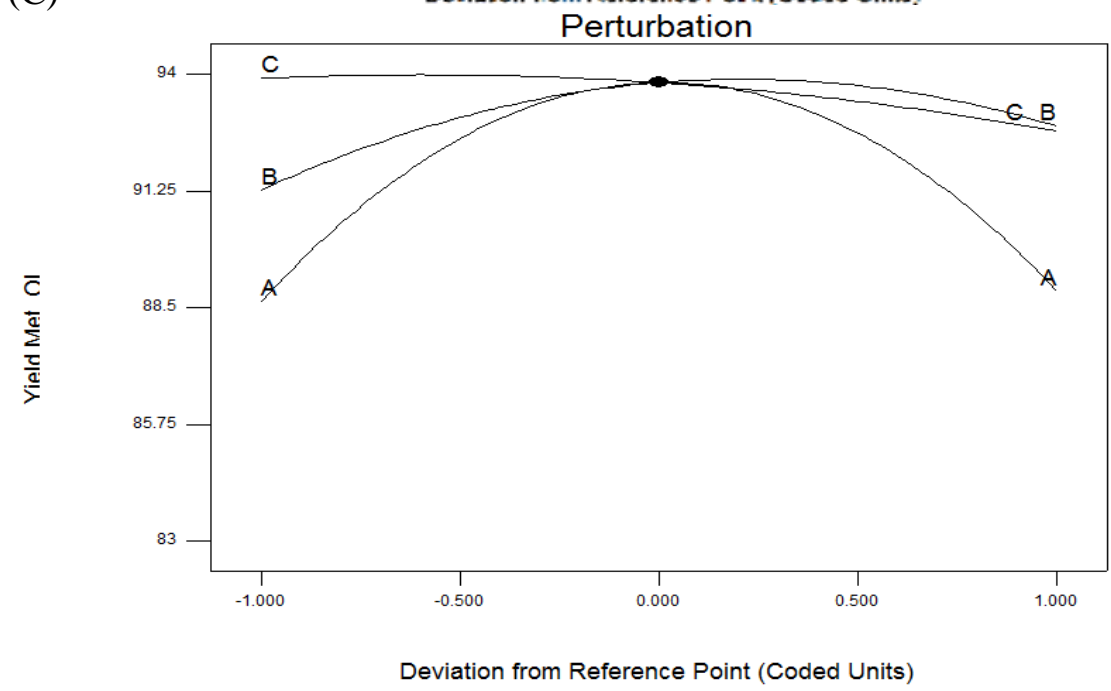

Fig 4: Perturbation plot showing effect of molar ratio of methanol/oleic acid, catalyst loading and reaction time on a) conversion of PFAD/oleic; b) methyl oleate; c) methyl palmitate yield. 


\section{Interaction of Process Variables on Responses}

The graphical representations of the model equations (3D response curves), were generated to study the influence of each process variable on the responses for the esterification of PFAD/oleic acid mixed. These are shown in Figs 5 -7. Fig 5 depicts the effect of the interaction $(\mathrm{AB})$ between molar ratio and catalyst loading on converion of PFAD/oleic acid, and both yields of methyl oleate and methyl palmitate. It is seen that $A B$ had a great significance on the responses. However, molar ratio had a negative effect in the quadratic equation. As a result, the conversion and yields increased with increase in methanol to PFAD/oleic acid molar ratio.

(a)

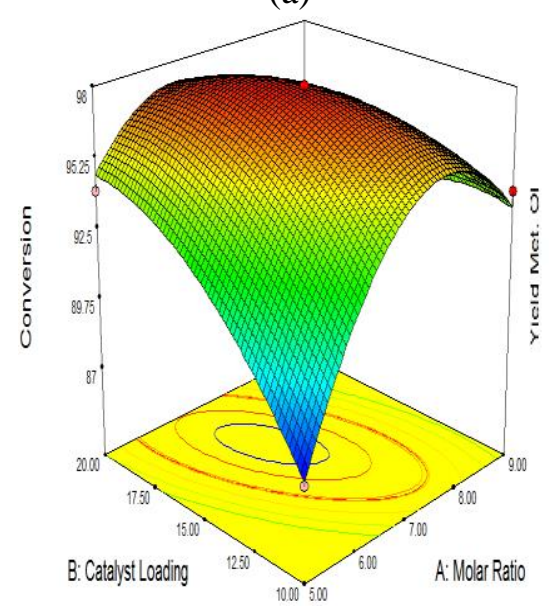

(b)

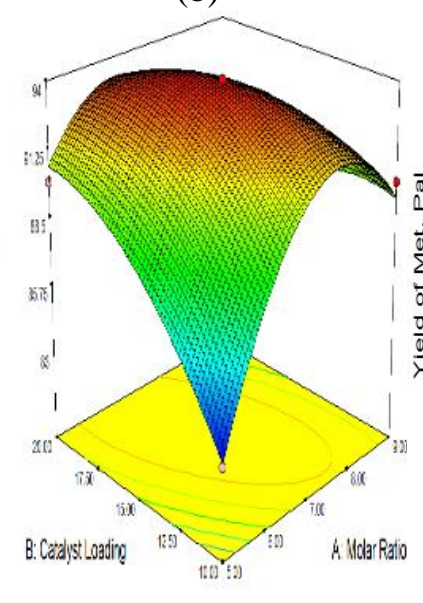

And the highest conversion and yields for PFAD/oleic acid was $97 \%$ at molar ratio 1:7 and catalyst loading $15 \mathrm{wt} \%$. While $92 \%$ and $94 \%$ yield were obtained at the same molar ratio and catalyst loading $f$ or methyl oleate and methyl palmitate respectively. Further increase in molar ratio led to a decrease in conversion and yield. A required amount was needed for the reaction to reach equilibrium, after which the excess methanol may lead to the flooding of active sites of the catalyst, and hinder the protonation of PFAD/oleic acid at the active sites [14]. In addition, excess methanol may introduce more water into the reaction which in turn might react with the FAME and convert it back to PFAD/oleic acid.

Fig 5:Response surface plots as a function of methanol to PFAD/oleic acid molar ratio and catalyst loading for a) PFAD/oleic acid conversion, b) methyl oleate yield and c) methyl palmitate yield.

The influence of methanol to PFAD/oleic acid molar ratio, and time on the responses are depicted in Fig 6. It can be seen that reaction time had a large significance on the reaction. The quadratic coefficients were negative in the quadratic equations (3.1-3.3). As a result, the conversion and yields proportionally increased and decreased with molar ratio and time respectively. Conversion and yields decreased for shorter reaction time, and high molar ratio of methanol to PFAD/oleic acid. And also at lower molar ratio and longer reaction time. There was an increase in conversion and yields from the start until $4.50 \mathrm{~h}$ and maintained after that. Conversions of $90 \%$ were obtained in the first two runs. However, a steady decline in conversion was obtained for the subsequent recycling. The highest conversion was obtained at $88 \%$, while the lowest was $60 \%$. [7] reported a similar trend in which the conversion increased till it reached $2 \mathrm{~h}$. The conversion remained unchanged till it reached $6 \mathrm{~h}$ before decreasing. This could be a result of a reverse in reaction (i.e. hydrolysis of esters) as an extended time favours the reverse reaction,[9]. Hence, the reduction in conversion and yields of methyl oleate and methyl palmitate. 
(a)

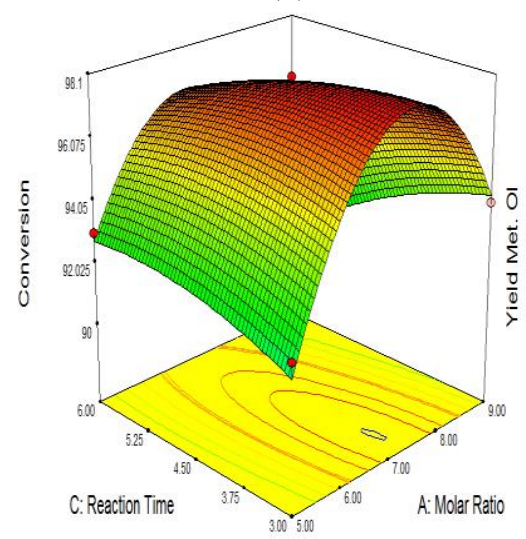

(b)

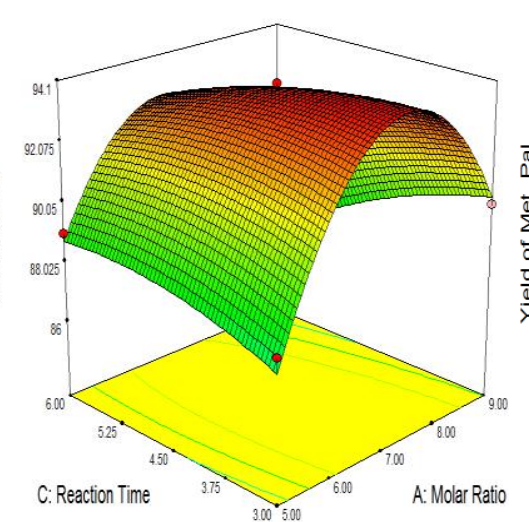

(c)

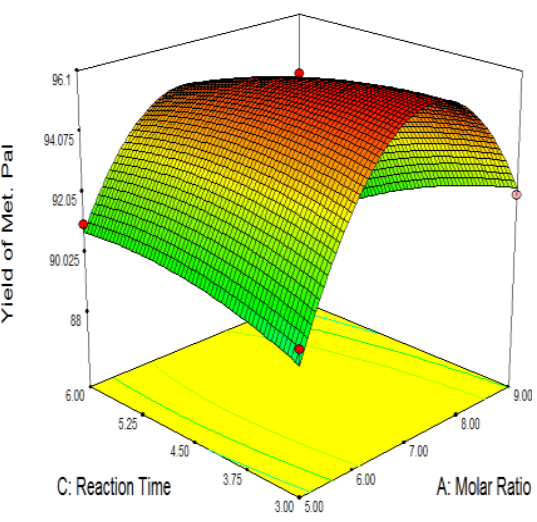

Fig 6:Response surface plots as a function of methanol to PFAD/oleic acid molar ratio and reaction time for a) PFAD/oleic acid conversion, b) methyl oleate yield and c) methyl palmitate yield.

Fig 7 shows the interaction between catalyst loading (B) and time (C). The effect of the interaction was clearly demonstrated in the graphs. For short reaction times, the catalyst loading had high significance in increasing the conversion of PFAD/oleic, and yield of both methyl oleate and methyl palmitate. However, the effect of catalyst loading decreased as the reaction time increased. For $4.50 \mathrm{~h}$ reaction time, increasing the amount of catalyst from $10-15 \mathrm{wt} \%$ increased the conversion up to $97 \%$. However, there was a decrease in the conversion as the reaction time was increased beyond 5h. For short reaction times, the

(a)

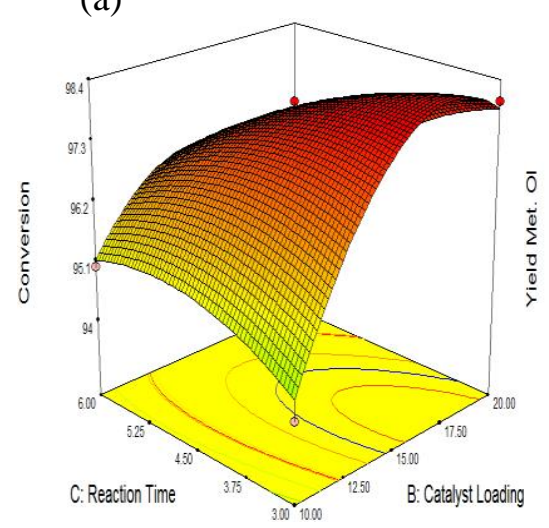

reaction is far away from equilibrium. Therefore, a sufficient amount of catalyst is needed for it to attain equilibrium, and obtain a high conversion and yield. However, prolonging the time after it has reached equilibrium is not necessary, and will only reduce the conversion and yield. This is due to the fact that the catalyst loading and time interaction gives a negative effect in the quadratic equations (3.1-3.3). Therefore, based on these results, a medium reaction time and high amount of catalyst is the best condition for the reaction. (b)

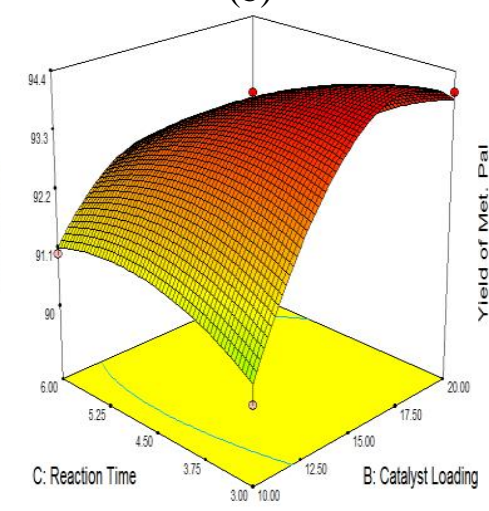

(c)

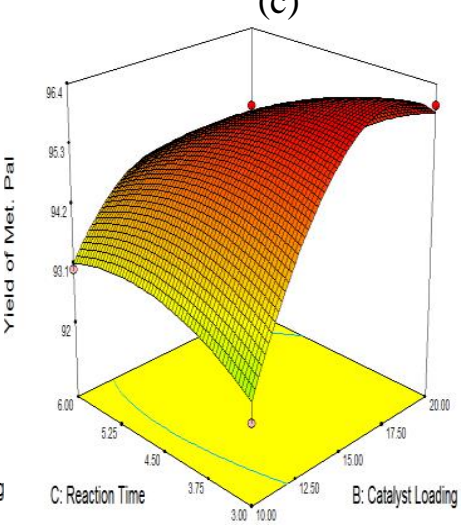

Fig 7: Response surface plots as a function of catalyst loading and reaction time on a) PFAD/ oleic acid conversion; b) methyl oleate yield; c) methyl palmitate yield

\section{CONCLUSION}

Response surface methodology showed a relationship between three variables that significantly affected the rate of conversions and yields of both feeds. Highest conversion obtained for PFAD/oleic acid, and yields (methyl oleate and methyl palmitate) were
$98 \%, 94 \%$ and $96 \%$ respectively. The linear terms of catalyst loading and reaction temperature were the most significant parameters that affected the responses. High conversions 
and yields were obtained by increasing the catalyst loading and reaction time, as shown in the response surface plots.

The synthesized sulfonated carbon showed a good stability after it was recovered successfully from the reaction mixture, and reused for the subsequent esterifications/ transesterifications of PFAD/oleic acid.

Finally, it is proposed that sulfonated carbon is a suitable catalyst for the synthesis of biodiesel from high free fatty acid content feedstocks, owing to its activity. It can be easily recovered and reused after the whole process. The catalytic performance exhibited by this catalyst, has great potential for biodiesel

\section{REFERENCES}

1 Alhassan, F.H., Yunus, R., Rashid, U., Sirat, K., Islam, A., Lee, H.V., TaufiqYap, Y.H. (2013). Production of biodiesel from mixed vegetable oils using Ferric hydrogen sulphate as an effective reusable heterogeneous solid acid catalyst. Appl. Catal. A - Gen. 456, 182-187

2 Association of Official Analytical Chemist (AOAC) (1999). Official method of analysis $15^{\text {th }}$ Edition Washington DC, .12-13

3 Bezerra, M. A., Santelli, R. E., Oliveira, E. P., Villar, L. S., Escaleira, L.A. (2008). Response Surface Methodology (RSM) as a Tool for Optimization in. Analytical Chemistry. Talanta. 76(5), 965-977

4 Cho, H.J, Kim J K Ahmed, F., Yeo,Y.K (2003). Life - cycle greenhouse gas emission and energy balances of a biodiesel production from palm fatty acid distillate (PFAD) Appl. Energy 111, 470 -488.

5 Dawodu, F.A.; Ayodele, O.; Xin, J.; Zhang, S.; Yan, D (2014). Effective conversion of non-edible oil with high free fatty acid into biodiesel by sulphonated carbon catalyst. Appl. Energy 114, 819-826

6 Dwived G, and Sharma M. P (2005). Application of Bos- Brhnken design in optimization of biodiesel yield from Pongamia oil and its stability analysis Fuel 145,256 -262

7 Elsheikh, Y. A., Man, Z., Bustam, M. A., Yusup, S. and Wilfred, C.D. production from feedstocks containing high free fatty acid content, upon some modifications.

\section{ACKNOWLEDGEMENT}

The authors are gratefull to the Department of Chemical and Energy Engineering,Universiti Technology Malaysia for allowing us to conduct this reseach and Prof..Dr Nor Aishah Saidina Amin for her continuous support,assistance, patience,motivation and endless guidance during the course this research

(2011). Brønsted Imidazolium Ionic Liquids: Synthesis and Comparison of Their Catalytic Activities as PreCatalyst for Biodiesel Production through Two Stage Process. Energy Conversion and Management. 52(2), 804-809.

8 Gui, M. M., Lee, K.T., Bhatia, S. S. (2009). Supercritical ethanol technology for the production of biodiesel: process optimization studies. J. Supercrit. Fluids. 49, 286292.

9 Leung D. Y. C., Guo, Y. (2006). Transesterification of Neat and Used Frying Oil : Optimization for Biodiesel Production. Fuel Processing Technology. 87 (10), 883-890.

10 Lokman, I. M., Rashid, U., TaufiqYap, Y. H.,Yunus, R. (2015). Methyl ester production from palm fatty acid distillate using sulfonated glucosederived acid catalyst. Renewable Energy. 81, 347-354.

11 Lokman, I. M., Rashid, U., TaufiqYap, Y. H. (2015). Production of Biodiesel from Palm Fatty Acid Distillate using Sulfonated-Gluc ose Solid Acid Catalyst: Characterization and Optimization. Chinese Journal of Chemical Engineering. 23(11), 18571864.

12 Islam, A., Taufiq-Yap, Y.H., Chu, C.M., Chan, E.-S., Ravindra, P (2013). Studies on design of heterogeneous catalysts for biodiesel production. Process Saf. Environ. 91, 131-144

13 Rashid, U., Anwar, F., Ashraf, M., 
J. Chem. Soc. Nigeria, Vol. 46, No.1, pp 0036 - 0048 [2021]

Saleem, M., Yusup, S. (2011). Application of response surface methodology for optimizing transesterification of Moringa oliefera oil: Biodiesel production. Energy Convers. Manag. 52, 3034-3042.
14 Zhang Y ,Wong ,W and Yung,K (2014).Biodiesel production via esterificatio of oleic acid catalyzes by chlorosulfonic acid modified Ziconia.Applied Energy.116,191 -198. 